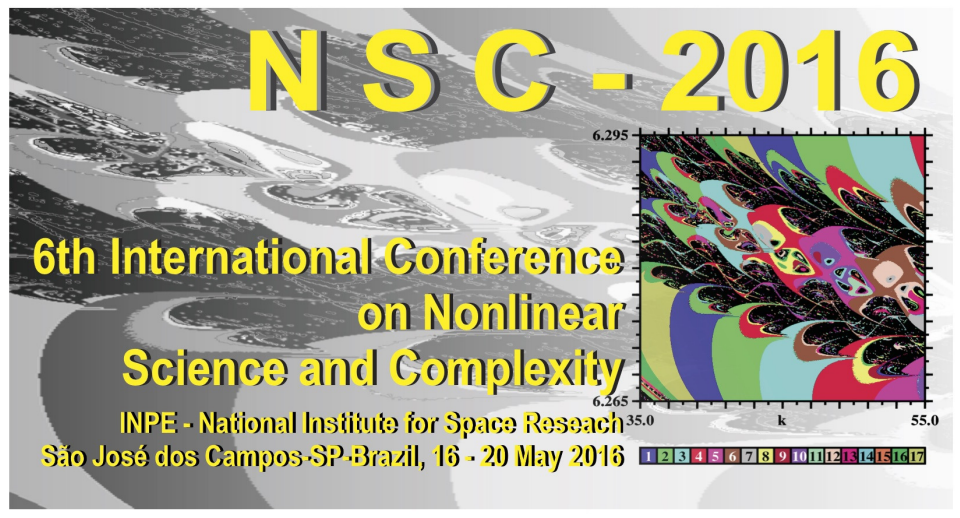

INPE - National Institute for Space Research

São José dos Campos - SP - Brazil - May 16-20, 2016

\title{
DYNAMICAL CHARACTERIZATION OF NONLINEAR SYSTEMS THROUGH COMPLEX NETWORKS
}

\author{
Juliana C. Lacerda ${ }^{1}$, Vander Luis de Souza Freitas ${ }^{2}$, Elbert E. N. Macau ${ }^{3}$ \\ ${ }^{1}$ Msc candidate on Applied Computing at INPE, São José dos Campos, Brazil, juliana.lacerda@inpe.br \\ ${ }^{2} \mathrm{PhD}$ candidate on Applied Computing at INPE, São José dos Campos, Brazil, vander.freitas@inpe.br \\ ${ }^{3}$ Associated Laboratory for Computing and Applied Mathematics (LAC) - INPE, Sao Jose dos Campos, Brazil, elbert.macau@inpe.br
}

\begin{abstract}
In this work, we present an approach for the dynamical characterization of a nonlinear system using symbolic dynamics and complex networks. As an example, we apply the proposed methodology to the Logistic Map. Some properties of the networks generated by the time series of this map match with the expected behaviors of the system. In other words, our methodology could identify periodic windows and chaos.
\end{abstract}

keywords: Chaos and Global Nonlinear Dynamics, Complex networks, Time Series Analysis, Logistic Map.

\section{INTRODUCTION}

The dynamical characterization of nonlinear systems is a great challenge and it is usually performed by using Lyapunov exponents and bifurcation diagrams, which is not always an easy task. The contribution of this work is to provide an alternative characterization using a combination of symbolic dynamics and the formalism of complex networks.

The methodology is exemplified in the context of the Logistic map:

$$
x_{n+1}=r x_{n}\left(1-x_{n}\right)
$$

where $0 \leq x_{n} \leq 1$ is a dimensionless measure of the population in the nth generation and $0 \leq r \leq 4$ is the growth rate.

R. May [1] showed that this model presents a "complex" behavior when its parameter space is explored. Feigenbaum [2] took this model as a paradigm to show the now called "period-doubling route" to chaos, displaying the universal behaviors governing dynamic transitions. All these features make this model as a paradigm for a system that presents transition to a chaotic dynamics.

In complex networks, a network corresponds to a graph, which is composed by vertices connected by edges. We can represent relationships with this network (where the vertices represent entities and the edges represent the interactions between these entities) and analyze them by means of the theory of complex networks. Examples of networks include the internet, social networking, telephony, etc. [3,4].

In this work, we will make use of symbolic dynamics to allow us to apply the complex network formalism to analyze time series from the logistic map that are associated with different dynamical regimes of this system. In order generate the network, first we make a conversion of this time series to a binary series and then we make a conversion of this binary series into a decimal series. The network is built from this decimal series and its properties will be studied according to the formalism of complex networks. As a result, we have a robust and reliable technique, whose implementation does not require intense computational resources.

\section{METHODS}

One way to represent the time series from the Logistic map into networks is by converting the values through the computation of the Top Integral Function $[5,6]$ as follows:

$$
Y_{k}=\left[N . x_{k}\right]=\min \left\{i \in Z \mid N . x_{k} \leq i\right\}
$$


in which $Y_{k}$ is the integer associated to the value $x_{k}$ of the series, $N$ is the number of vertices of the network and $i$ is the smaller value such that $N . x_{k} \leq i$. In this case, each $Y_{k} \in$ $[1, N]$ corresponds to a vertex in the network. As shown in [5], the characteristics of the generated network express the Logistic map properties.

In this work we propose an approach that uses symbolic dynamics. For each point of the time series generated by the Logistic map we assign the symbol 0 , if the value is between $[0,0.5]$, or 1 otherwise. After that, this new series is visited using a moving template of $M$ bits, which will slide from the beginning to the end one position at a time. The $M$-bit templates are binary numbers that will be converted to decimal numbers, which will form the decimal series we want for the network representation. We can see how this process works in Figure 1.

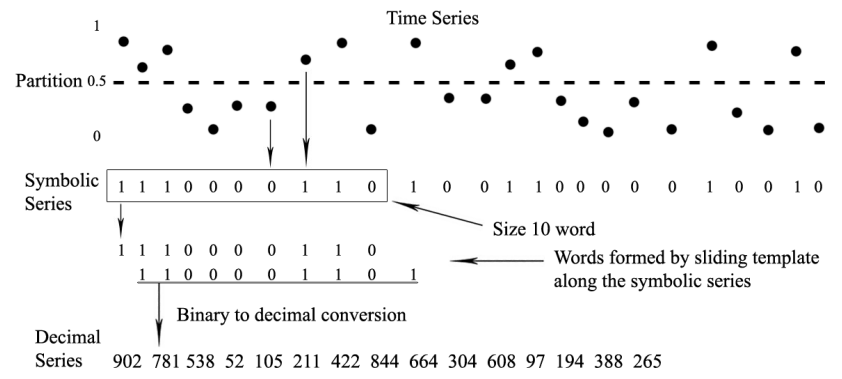

Figure 1 - How to generate the symbolic and decimal series from the Logistic map time series using a 10-bit template.

Each value of the decimal series corresponds to a node in the network. The connections among nodes occur sequentially, with nodes being linked to their adjacent neighbors in the decimal series. Since the template has $M$ bits, the network will have $2^{M}$ nodes. Loops are allowed and there can exist only one edge connecting a pair of nodes [5]. In Figure 2 there are some examples of networks generated from the Logistic map, where we can see the existence of stable periodic windows. To make sure they corresponds to truly periodic windows, all the nodes must form a single cycle. When we have period 1 , the decimal series has always the same value, so there is only one node at the network (which is connected to itself). When there is period 2 the decimal series has two repeating values, so we have two nodes connected by one edge. In this case the average degree of the network will be 1 . For periods bigger than 2 it is possible to see very well defined cycles resulting in graphs with average degree equal to 2 .

\section{RESULTS}

We performed experiments with 10-bits templates, i.e., using networks of $N=2^{10}$ nodes. The total number of iterations of the Logistic map was 10500 (discarding the first 500 ) and we used a set of 1000 values of $r$ ranging between 2.9 and 4.0 , thereby generating a total of 1000 networks. The initial condition was fixed to $x_{0}=0.7$, since the the results

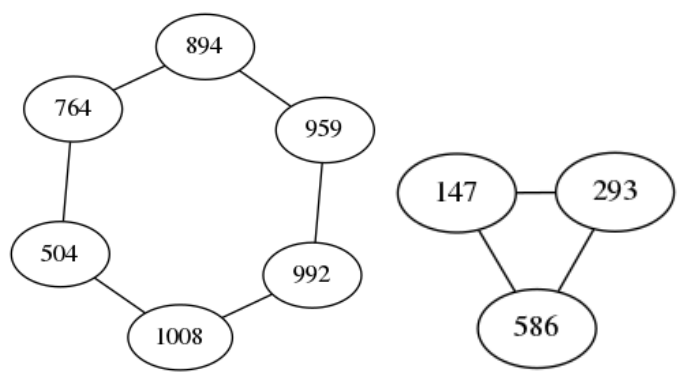

(a) $r=3.629$ : Period 6 .

(b) $r=3.835$ : Period 3 .

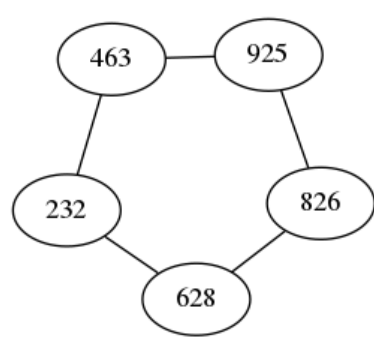

(c) $r=3.906$ : Period 5 .

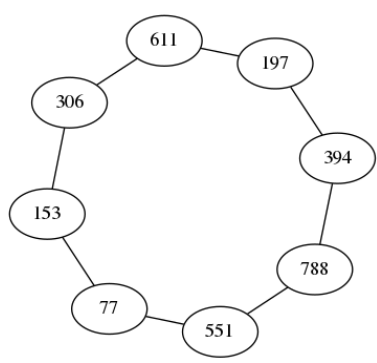

(d) $r=3.961$ : Period 8 .
Figure 2 - Networks generated from the time series of the Logistic map for several Logistic parameters $r$.

are nearly the same for different initial conditions [6].

In the upper part of Figure 3 we have the degree of the vertices as a function of the logistic parameter $r$. On the vertical axis we have the 1024 vertices of each network and in the horizontal axis we have the logistic parameter. The degrees are represented by color, with the lowest being blue and yellow being the highest. In the middle of Figure 3 we have the Lyapunov exponents $(\lambda)$, which measures the exponential divergence between orbits over time. Negative Lyapunov exponents indicate the absence of chaos and the existence of periodic windows. At the bottom of the figure we plotted the bifurcation diagram of the Logistic map to emphasize the meaning of the Lyapunov exponents.

The betweenness centrality of a vertex measures the number of shortest paths that pass through that vertex. So a vertex with high betweenness has a great importance for the network. The betweenness centrality of a vertex is given bellow

$$
b(v)=\sum_{s \neq v \neq t} \frac{\sigma_{s t}(v)}{\sigma_{s t}}
$$

where $\sigma_{s t}$ is the total number of short paths between vertices $s$ and $t$ and $\sigma_{s t}(v)$ is the number of these paths that pass through $v$. The normalized betweenness centrality of a vertex $v$ is

$$
\operatorname{norm}(b(v))=\frac{b(v)-\min (b)}{\max (b)-\min (b)}
$$

where $b$ is a vector that contains the value of the betweenness centrality of all vertices of the network, $\min (b)$ and $\max (b)$ are, respectively, the minimum and maximum values of $b$. As 

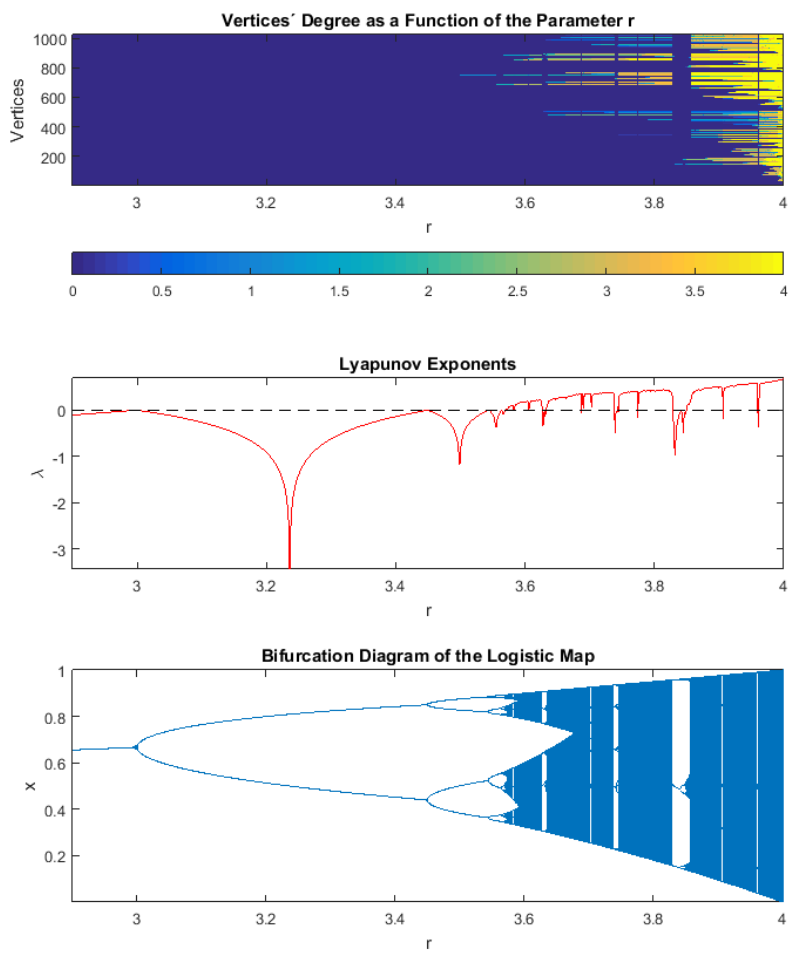

Figure 3 - From top to bottom: Vertices' degree, Lyapunov exponents and bifurcation diagram of the Logistic map. The horizontal axis is the parameter $r$.

we are working with a thousand networks, we will consider the average of the normalized betweenness centrality.

The density of a network $(D)$ is its number of edges $(E)$ over the maximum possible number of edges,

$$
D=\frac{E}{N(N-1) / 2}
$$

in which $N$ is the number o nodes.

Bellow, in Figure 4, we have the number of connected vertices and the density of the network, followed by the diameter, average degree, mean normalized betweenness centrality and Lyapunov exponents, all of them as functions of $r$.

\section{DISCUSSION}

In the upper part of Figure 3 we can see, starting from $r=3.5$, some periodic windows (blue vertical strips) for the same values of the Logistic parameter where there are windows at the bifurcation diagram and negative Lyapunov exponents.

The number of connected vertices and the density grow as $r$ increases (Figure 4), but there is a sudden drop where the Lyapunov exponents are negative. In these regions we have periodic windows so that a few vertices are connected and the number of connections between them is low. For $r=4$, practically all vertices are connected and the number of connections between them is higher, which is expected
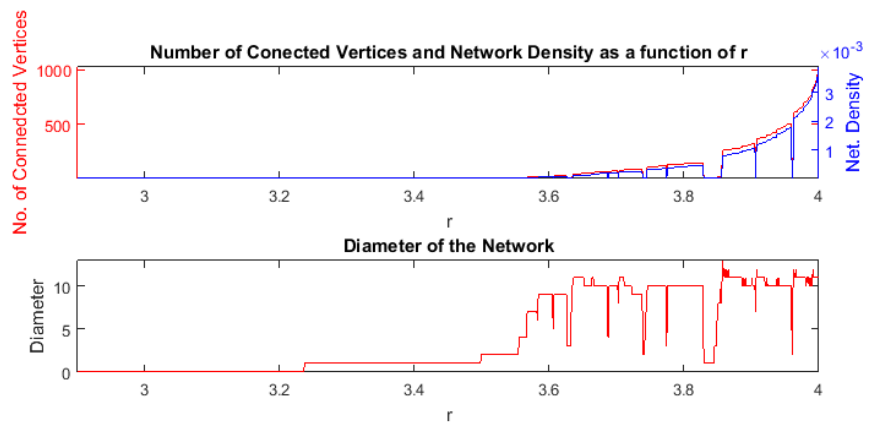

Average Degree

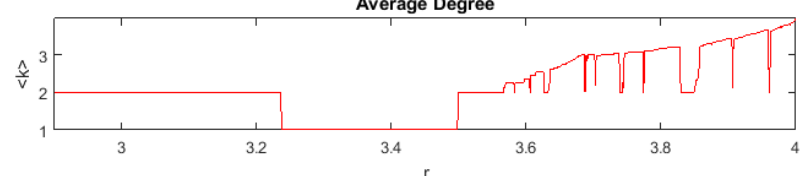

Mean Normalized Betweenness Centrality
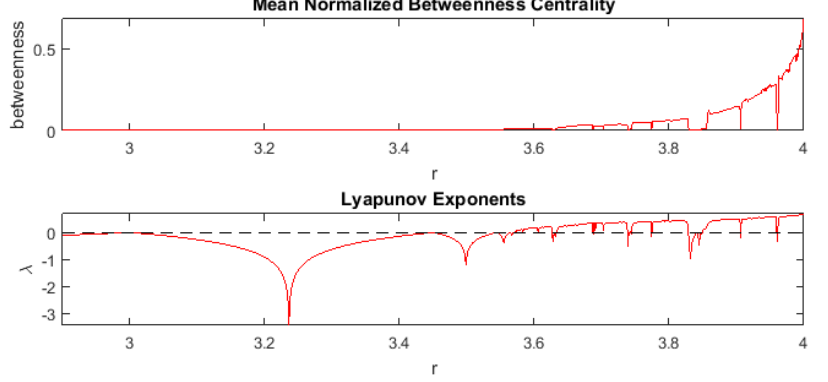

Figure 4 - From top to bottom: Number of connected vertices (red) and the density of the network (blue), diameter, average degree, mean normalized betweenness centrality and Lyapunov exponents as a function of $r$.

because of the presence of chaos. Notice that the density is always low, in the order of $10^{-3}$, which means that the networks generated by this method are sparse.

The following measures of diameter, average degree and mean normalized betweenness centrality have several "drops" for the same values of the logistic parameter $r$ where we can observe negative Lyapunov exponents and therefore when there are periodic windows. In these windows, as previously mentioned, we have networks that form cycles of various periods, as shown in Figure 2.

The largest of the smaller paths between all vertices of a network (diameter) tends to be lower when this network has a cycle, so the diameter of these networks tend to be smaller than the diameter of networks representing chaos. Also, the average degree of networks corresponding to periodic windows tend to be 2 , since they form cycles as those presented in Figure 2. The number of smaller paths passing through a given vertex decreases when there is a cycle, which causes the average betweenness centrality to be smaller.

Using only the graphics of the diameter, average network degree, number of connected vertices or density of the network, it is possible to predict the existence of the following periodic windows: $r=$ 3.583, 3.606, 3.629, 3.687, 3.703, 3.740, 3.774, 3, 835, 3, 906 , 3,961 . For the average of the normalized betweenness cen- 
trality, this correspondence was obtained from $r=3,687$.

We can also observe that at the chaotic regime, the diameter of the networks is around 10 (about $1 \%$ of the number of nodes) and the average degree is between 3 and 4 . As the average degree is low, one can say that the networks are sparse (as previously mentioned).

\section{CONCLUSION}

We generated time series for many values of the Logistic parameter $r$. Then we built new representations of the series by applying symbolic dynamics techniques. From these results we generated networks, each of them representing one value of $r$, and we found correspondences between their properties, the Lyapunov exponents and the bifurcation diagram. Therefore, our methodology was able to predict the existence of chaos and the appearance of some periodic windows of the Logistic map. In future works we will apply this methodology to other maps and also to continuous systems.

\section{ACKNOWLEDGMENTS}

J.C.L. was partially supported by CAPES; V.L.S.F. was partially supported by CNPq and CAPES. E.E.N.M. would like to thank FAPESP project 2011/50151-0.

\section{References}

[1] R. M. May, "Simple mathematical models with very complicated dynamics", Nature, Vol. 261, pp. 459467, 1976.

[2] M. J. Feigenbaum, "Universal behavior in nonlinear systems", Physica D: Nonlinear Phenomena, Vol. 7, pp. 16-39, 1983.

[3] A. L. Barabasi e R. Albert, "Emergence of scaling in random networks", Science, Vol.286, No. 5439, pp. 509-512, 1999

[4] S. Boccaletti et al, "Complex networks: Structure and dynamics", Physics Reports, Vol. 424, No. 4-5, pp. 175-308, 2006

[5] V. L. S. Freitas e E. E. N. Macau, "Uso de redes complexas para caracterização dinâmica de sistemas não lineares", Proceeding Series of the Brazilian Society of Computational and Applied Mathematics, (to appear).

[6] X. Yu, Z. Jia and X. Jian, "Logistic mapping-based complex network modeling", Applied Mathematics, Vol. 4, pp. 1158-1562, 2013. 